\title{
Torsion in a massive hematosalpinx with a functional rudimentary horn: a rare cause of acute abdomen in adolescence, managed laparoscopically.
}

\author{
Arpita De ${ }^{1 *}$, Renu Misra², Abhinav Jain ${ }^{3}$
}

\begin{abstract}
${ }^{1}$ Department of Obstetrics and Gynecology, ${ }^{3}$ Department of Radiodiagnosis Hamdard Institute of Medical Sciences and Research, Hamdard Nagar, New Delhi, India

${ }^{2}$ Department of Obstetrics and Gynecology, Sitaram Bhartia Institute of Medical Sciences and Research, New Delhi, India
\end{abstract}

Received: 06 September 2020

Accepted: 08 October 2020

\section{*Correspondence:}

Dr. Arpita De,

E-mail: arpitade07@gmail.com

Copyright: () the author(s), publisher and licensee Medip Academy. This is an open-access article distributed under the terms of the Creative Commons Attribution Non-Commercial License, which permits unrestricted non-commercial use, distribution, and reproduction in any medium, provided the original work is properly cited.

\begin{abstract}
Congenital anomalies of the female genital tract may remain undiagnosed till adolescence. Unicornuate uterus with a rudimentary horn is rare and found in 1 in 100000 women. A functional rudimentary horn may cause hematometra, hematosalpinx, endometriosis and its complications. This is a case of a 13-year-old girl who had a hematometra in a functional rudimentary horn and a hematosalpinx with torsion giving rise to an acute abdomen and a 16-week mass abdomen. Laparoscopy was done followed by detorsion, salpingectomy and transection of the rudimentary horn. This article should sensitize the need to correct the anomaly while preserving the reproductive potential of the girl. A review of the salient points of laparoscopy in such cases have been further discussed.
\end{abstract}

Keywords: Acute abdomen in adolescent girls, Unicornuate uterus with functional rudimentary horn, Laparoscopic rudimentary horn excision.

\section{INTRODUCTION}

The prevalence of a unicornuate uterus with rudimentary horn is very rare and occurs in 1 in $100,000 .{ }^{1}$ When there is a functional endometrium in a pubertal girl then complications like hematometra, hematosalpinx, endometriosis and torsion of the fallopian tube can occur. Torsion is very rare in the 1-20 years age group with an approximate prevalence of $0.005 \%$ compared to $2.5-7.5 \%$ in the adult population. ${ }^{2}$

This is a very rare case of torsion occuring in a case of hematosalpinx with hematometra in a functional rudimentary horn in a 13-year-old girl which has been successfully managed laparoscopically. The tips of laparoscopic management have been further discussed here.

\section{CASE REPORT}

A 13-year-old girl presented to the OPD with acute pain abdomen since the last one day and a history of mild to moderate pain lower abdomen since the last three months. She had menarche one year back and used to have severe dysmenorrhoea. One ultrasound done outside showed a $12 \times 8 \mathrm{~cm}$ mass with echogenic contents, arising from the left adnexa along with hematometra. However, the diagnosis was not definitive. On examination she had a pulse rate of $100 \mathrm{bpm}, \mathrm{A}$ BP-100/66 and tenderness and guarding of the abdomen. A 16 weeks soft, tender mass was palpable.

MRI revealed a bicornuate uterus with blood clots within the left half. There was a large coiled tubular structure of around $11.4 \times 11.8 \mathrm{~cm}$ with blood clots which was 
consistent with a hematosalpinx and it showed torsion (Figure 1, 2).

The right horn was communicating with the cervix and the right sided adnexa was normal. Both kidneys and ureters were normal.

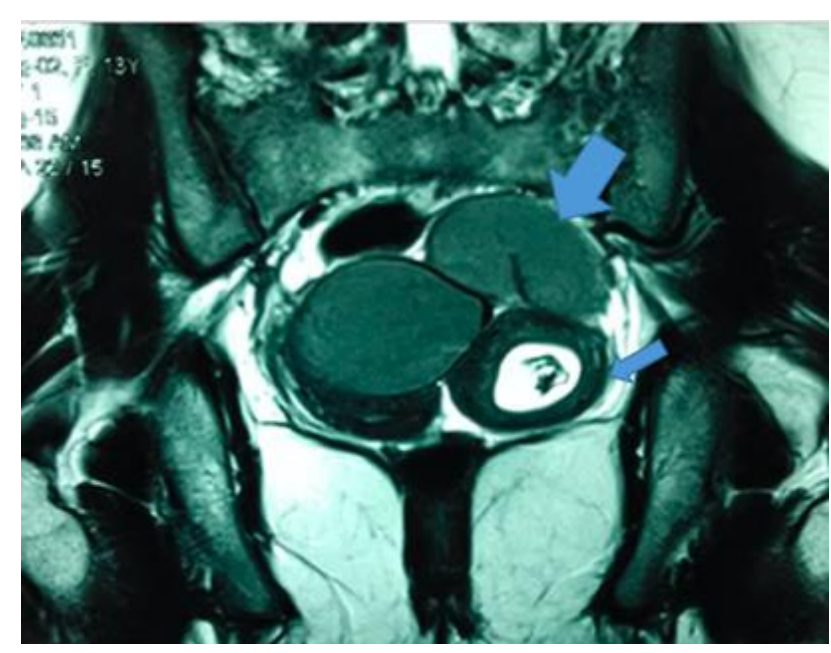

Figure 1: MR Pelvis showing bicornuate uterus with left hematometra and hematosalpinx.

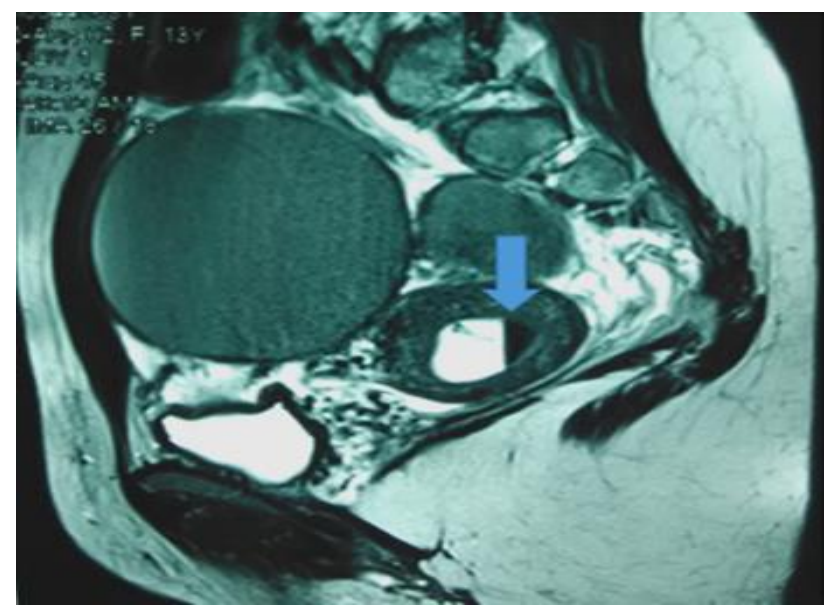

Figure 2: MRI Pelvis showing dilated endometrial cavity with fluid level suggesting hemorrhagic contents.

On emergency laparoscopy there was a huge cystic mass covering the pelvis. This mass was darkish blue and had adhesions all around it- primarily with the omentum and the abdominal wall. Aspiration was done and around $30^{\circ} \mathrm{c}$ of dark coloured fluid was drained out. After further adhesiolysis, another mass of $5 \times 4 \mathrm{cmwas}$ seen, which was identified as left sided rudimentary horn. It had the fibrosed thickened round ligament, left tube and ovary attached on the left cornual end. The horn was soft and boggy. The cystic mass initially encountered turned out to be the dilated left fallopian tube with hematosalpinx and torsion of three turns. The torsion was corrected and the tube was found to be necrotic, gangrenous and had bleeding from the fimbrial edge. Left sided salpingectomy was done. To conserve the ovarian vessels the salpingectomy was done close to the tube. Then the left utero-ovarian pedicle was transected with a ligasure vessel sealing device. The utero-vesical pouch was opened and the bladder pushed down. This was sufficient to expose its base and there was no need for uterine artery ligation from its origin. The accessory horn was excised with $10 \mathrm{~mm}$ ligasure and the plane was found to be between the two myometrium without any breach of cavity.

On the cut section the accessory horn was found to be full with old blood clots and without any communication with the cervix. This confirmed the final diagnosis of Unicornuate uterus with functional noncommunicating left horn. The postoperative period was uneventful and the abdominal pain and dysmenorrhoea disappeared completely. She was fine till the last follow up which was two years post-surgery.

\section{DISCUSSION}

Unicornuate uterus comprises of around 5\% of Mullerian anomalies. ${ }^{3}$ It occurs when there is partial or complete failure of development of the Mullerian duct on either side. The Unicornuate uterus may be solitary (hemiuterus) or may have an accessory horn, which may or maynot be communicating to the cervix. $75-90 \%$ of Unicornuate uterus with rudimentary horns are of the noncommunicating types. Unicornuate uterus with a noncommunicating horn can be classified as $\mathrm{U} 4 \mathrm{aCOVO}$ (recent ESHRE-ESGE classification) or as class II b ( by the traditional, but more commonly used AFS classification). ${ }^{4,5}$ While the ESHRE classification only mentions a unicornuate uterus- with or without rudimentary horn, the more traditional AFS further classifies the rudimentary horn into three categories- with or without a communicating cavity and the third with no cavity in the horn. In this instance the AFS enables a complete preoperative diagnosis which helps in planning the management of the patient. A solitary unicornuate uterus or a horn with no cavity doesn't require any surgery, while the other types of horns always need to be surgically resected. Functional horns, if left, can lead to hematometra, hematosalpinx and endometriosis in due course of time. Such women may have chronic pain abdomen, dysmenorrhoea, dyspareunia, subfertility and pregnancy complications like abortions, ectopic pregnancies, cervical incompetence, growth retardation, preterm labour and many others. ${ }^{6}$

The acute pain may be due to hematometra or hematosalpinx or torsion, which if not operated soon would compromise the tubo-ovarian viability. Torsion in the adolescent age group could be attributed to an enlarged utero-ovarian ligament or Mullerian anomalies as there are abnormal connections between the ovary and other structures. $^{7,8}$ 


\section{Diagnosis}

For any young girl with acute on chronic pain abdomen and mass abdomen, pregnancy should be ruled out. Sonography may be diagnostic but one may require a 3-D sonography or MRI for the complete diagnosis. Workup should also include a thorough assessment of the renal and ureteric systems as $40 \%$ of the unicornuate uterus are associated with renal agenesis or a pelvic kidney. In cases of acute abdomen, it is absolutely essential to do a doppler to rule out torsion. MRI may show thickened pedicle, enlarged and superiorly pushed ovary with increased signal in the ovarian stroma. ${ }^{9}$ The thickness of the myometrium of the horn and its distance from the cervix should be noted (to assess the need for Uterine artery ligation). Long standing cases may be associated with extensive adhesions which may require skilled surgeons.

\section{Role of endoscopy}

For young girl's laparoscopy is recommended, while in sexually active women hystero-laparoscopy is recommended. Hysteroscopy helps in diagnosis of vaginal or cervical duplication, which may be missed out otherwise. Delay in surgery in cases of torsion may permanently compromise the ovarian function. Torsion may occur in the complete tubo-ovarian pedicle or in the tube alone (as in this case of hematosalpinx) or may involve the rudimentary horn itself. Torsion usually occurs in gravid rudimentary horns. ${ }^{10}$

Few similar cases with bicornuate or hemi uterus with noncommunicating accessory horn with acute abdomen have been reported in the literature. Transection of the horn is recommended by all. ${ }^{1,11-16}$

\section{Tips during laparoscopic surgery in such cases}

1) Extensive adhesiolysis may be required to delineate the complete anatomy. Nature of the mass, the normal uterus and the rudimentary horn should be correctly identified before commencement of the transection. Location of bilateral tubes, ovaries, round ligament and uterosacrals help in the diagnosis. In parous women, hysteroscopy helps in the diagnosis. Ultrasonic shears or vessel sealing bipolar devices will be required in such cases 2) torsion should be managed conservatively whenever possible. Detorsion may be followed by ovariopexy (especially if it occurred in a normal sized tube and ovary) ${ }^{9}$ 3) look for signs of endometriosis and ablation of lesions to relieve symptoms. Cystectomy for small sized endometriomas should not be done, especially in young women to conserve ovarian tissue for further reproductive and hormonal function. 4) retroperitoneum may be opened. This will enable visualisation of the ureter, as aberrant ureteric courses are more common in mullerian anomalies. Further, the Uterine artery may be ligated at its origin to enable bloodless transection of the horn. In case there is a visible fibrous band, uterine artery ligation may not be required. 5) the transection can be done with bipolar vessel sealors (like ligasure $10 \mathrm{~mm}$ ) or surgical energy device like thunder beat or with a monopolar hook. In parous women the hysteroscopic transillumination helps in assessment of the plane of transection. Hemostasis may require suturing in some cases. It is advisable to cover the sutures with adhesion barriers. 6) the horn may be taken out by in bag morcellation or by piecemeal excision through an enlarged suprapubic incision.

\section{CONCLUSION}

Acute pain in a young girl with pre-existing chronic pain should raise the suspicion of Mullerian anomalies with or without torsion. MRI is the gold standard for diagnosis. Endoscopic surgery should be done by expert surgeons to reduce morbidity. Conservative management should be done in cases of torsion unless the tube is grossly damaged. The accessory horn with functional endometrium should be excised following the correct principles as mentioned.

\section{Funding: No funding sources \\ Conflict of interest: None declared \\ Ethical approval: Not required}

\section{REFERENCES}

1. Rani A, Kumari M, Shipra. A Case of Noncommunicating Uterine horn containing Functional Endometrium. Gynecol Obstet. 2015;5:9.

2. Guthrie BD, adler MD, powell EC. Incidence and trends of pediatric ovarian torsion hospitalizations in the United states, 2000-2006. Pediat. 2010;125:5328.

3. Reichman D, Laufer MR, Robinson BK. Pregnancy outcomes in unicornuate uteri: A Review. Fertil Steril. 2009;91(05):1886-94.

4. Chandler TM, Machan LS, Cooperberg PL, Harris AC, Chang SD. Mullerian duct anomalies: from diagnosis to intervention. $\mathrm{Br} \mathrm{J}$ Radiol. 2009;82(984):1034-42.

5. Grimbizis. The ESHRE/ESGE consensus on the classification of female genital tract congenital anomalies. Hum Reprod. 2013;28(8):2032-44.

6. Paul PG, Chopade G, Das T, Dhivya N, Patil S, Thomas M. Accessory cavitated uterine mass: a rare cause of severe dysmenorrhea in young women. $\mathbf{J}$ Minim Invas Gynecol. 2015;22(7):1300-3.

7. Celik A. Long-term results of conservative management of adnexal torsion in children. J Pediatr Surg. 2005;40:704-8.

8. Gupta N, Nigam A, Tripathi R, De A. Unilateral tubo-ovarian agenesis with contralateral adnexal torsion in a premenarchal girl. Brit Med J Case Rep. 2018.

9. Chandler TM, Machan LS, Cooperberg PL, Harris AC, Chang SD. Mullerian duct anomalies: from 
diagnosis to intervention. $\mathrm{Br} \quad \mathrm{J}$ Radiol. 2009;82(984):1034-42.

10. Grimbizis. The ESHRE/ESGE consensus on the classification of female genital tract congenital anomalies. Hum Reprod. 2013;28(8):2032-44.

11. Nigam A, De A, Jain A, Gupta N, Tripathi R. Ovarian torsion in a 4-year-old girl: A Rare occurrence. J Minim Invas Gynecol. 2020;27(2); 244-5.

12. Chundawat R, Rastogi R, Tak A. Torsion of gravid horn of bicornuate uterus: a rare case report. Int $\mathrm{J}$ Reprod Contracept Obstet Gynecol. 2016;5:2428-30.

13. Amara DP, Nezhat F, Giudice L, Nezhat C. Laparoscopic management of a noncommunicating uterine horn in a patient with an acute abdomen. Surg Laparosc Endosc. 1997;7(1):56-9.

14. Mabrouk M, Arena A, Zanello M, Raimondo D, Seracchioli R. Unicornuate uterus with noncommunicating functional horn: diagnostic workup and laparoscopic horn amputation. Fertil Steril. 2020;113(4):885-7.

15. Faller E, Baldauf JJ, Becmeur F, Lehn A, Akladios CY, Lecointre L. Laparoscopic Management of a Rudimentary Uterine Horn. J Minim Invasive Gynecol. 2018;25(5):769-70.

16. Jan H, Katesmark M, Ghai V. A Stepwise Approach to Laparoscopic Excision of a Noncommunicating Rudimentary Horn. J Minim Invas Gynecol. 2019;26(4):600-1.

Cite this article as: De A, Misra R, Jain A. Torsion in a massive hematosalpinx with a functional rudimentary horn: a rare cause of acute abdomen in adolescence, managed laparoscopically. Int J Reprod Contracept Obstet Gynecol 2020;9:5127-30. 